\title{
Effect of regularization parameters on geophysical reconstruction
}

\author{
Zhou Hui' ${ }^{1,2 *}$, Wang Zhaolei ${ }^{3}$, Qiu Dongling ${ }^{4}$, Li Guofa $^{1,2}$ and Shen Jinsong ${ }^{1,2}$ \\ ${ }^{1}$ State Key Laboratory of Petroleum Resource and Prospecting, China University of Petroleum, Beijing 102249, China \\ ${ }^{2}$ Key Laboratory of CNPC Geophysical Prospecting, China University of Petroleum, Beijing 102249, China \\ ${ }^{3}$ Research Institute, Bureau of Geophysical Prospecting, Hebei 072751, China \\ ${ }^{4}$ China University of Petroleum, Beijing 102249, China
}

\begin{abstract}
In this paper we discuss the edge-preserving regularization method in the reconstruction of physical parameters from geophysical data such as seismic and ground-penetrating radar data. In the regularization method a potential function of model parameters and its corresponding functions are introduced. This method is stable and able to preserve boundaries, and protect resolution. The effect of regularization depends to a great extent on the suitable choice of regularization parameters. The influence of the edge-preserving parameters on the reconstruction results is investigated and the relationship between the regularization parameters and the error of data is described.
\end{abstract}

Key words: Geophysical data, inversion, error of data, regularization, regularization parameters

\section{Introduction}

Geophysical inversion is an important topic in exploration geophysics (Shen et al, 2008; Wang et al, 2008; Wei et al, 2008; Yang et al, 2008). However, the reconstruction result may not be stable. The purpose of regularization is to overcome the instability in reconstruction caused by data perturbation. The key of the application of regularization is to construct a regularization operator and to determine regularization parameters. A simple and well-known regularization method is to suppose the model is globally smooth. However, an actual geological model consists of not only smooth regions, but also evident boundaries that are important characteristics of the model. In the reconstructed results of seismic or ground-penetrating radar data, the subsurface structure characteristics (such as interfaces, faults and discontinuities) and lithology characteristics (such as cavities, pinchings and lenticular bodies) are regarded to be boundaries. These boundaries need to be preserved in order to obtain high-resolution reconstruction images with clear background and boundaries. It is difficult to preserve the boundaries and avoid excessive smoothing in the effective application of the regularization method (Zhang and Yang, 2003). Conventional smoothing methods cannot overcome the conflict of suppressing noise and preserving edges (Liu and Zhao, 2000) since the noise and edges are both high frequency components in a reconstructed image. The conventional smoothing method deals with the noise and boundaries equally, i.e., it suppresses and smoothes the high frequency components. As a result, the noise is weakened,

*Corresponding author. email: huizhou@cup.edu.cn Received September 5, 2008 and at the same time the boundaries are blurred. On the other hand, if the boundaries are preserved, the ability to resist noise is lowered. In practical reconstructions, it is necessary to trade off edge preservation and noise suppression. In this paper, we used the edge-preserving method to reconstruct the subsurface structure.

Kunisch and Zou (1998) pointed out that the success and effectiveness of the application of the regularization method depends to a great extent on the proper selection of regularization parameters and the feasibility of the selection principle. It is clear from the published literatures that the selection schemes mainly are either prior or posterior (Zou et al, 2006). The prior scheme has sufficient theoretical basis; it is simple, but complicated if singularity value decomposition is used, and the hypothesis condition is hard to satisfy. As a result, it is mainly used in theoretical analysis and instruction. The posterior scheme's theoretical basis is slightly insufficient; its computation burden is large by the iterative method, but it is convenient and flexible to use. Therefore, it has high practical value.

The prior scheme determines the regularization parameters by defining the regularization parameters as functions of the error of data and by considering the error and structure of data under different theoretical hypotheses. The prior scheme has its value for theoretical analysis, but it is difficult to validate the application conditions. Therefore, the prior scheme is currently researched mostly by mathematicians, while the posterior scheme is studied and applied in practice. Several methods of the posterior scheme have been developed (Jin and Hou, 1996; Guo et al, 2006; Ding and Jin, 2006; Han and $\mathrm{Fu}, 2005$; Zhang et al, 2005), for example, (1) the method based on the deviation theory, such as Morozov deviation theory, generalized deviation theory, Arcangel principle, and 
generalized Arcangel principle, (2) the method based on searching, such as one-dimensional searching and genetic algorithms, (3) the method based on statistics, such as the maximum likelihood method, (4) the graphic method, such as L curve and ridge trace method, (5) other methods, such as Tikhonov optimal principle and the generalized cross check principle. Among these methods, some (e.g., graphic method) lack theoretical basis, some (e.g., Tikhonov optimal principle) have very limited effect, some (e.g., searching method) have very low efficiency, and most are iterative methods. If the reconstruction technique is iterative, the computational burden is very heavy. In addition, the description of inverse problems differs among the methods, and there are various existing regularization methods. Unfortunately, there is no general and effective method to determine regularization parameters. This paper tries to investigate the effect of edge-preserving parameters on reconstruction results and the relation between the parameters and error of data by theoretical analysis and numerical calculation, in order to establish a basis on which the edge-preserving parameters can be determined reasonably and efficiently. The inversion method used in this paper is the forward-backward time-stepping method (Takenaka et al, 2000; Zhou et al, 2007).

\section{Geophysical inversion method with edge- preserving regularization}

Generally, only one component of vibration or electric field is used in exploration (Wang et al, 2007) and thus in inversion. We derived the inversion equations for multiple components, but the reconstruction example in this paper was conducted by using one component. We constructed a cost functional $Q(\mathbf{p}, \mathbf{b})$ with a regularization term (Takenaka et al, 2000; 2001):

$$
Q(\mathbf{p}, \mathbf{b})=Q_{1}(\mathbf{p})+\boldsymbol{\lambda} \cdot \mathbf{Q}_{2}(\mathbf{p}, \mathbf{b}) \quad \lambda_{i} \geq 0
$$

where

$$
\begin{gathered}
Q_{1}(\mathbf{p})=\int_{0}^{c T} \sum_{m=1}^{M} \sum_{n=1}^{N} K_{m}\left(\mathbf{r}_{n}^{r}, t\right)\left|\mathbf{v}_{m}\left(\mathbf{p} ; \mathbf{r}_{n}^{r}, t\right)-\tilde{\mathbf{v}}_{m}\left(\mathbf{r}_{n}^{r}, t\right)\right|^{2} \mathrm{~d} t \\
Q_{2 i}(\mathbf{p}, \mathbf{b})=\varphi\left(\frac{\nabla p_{i}}{\chi_{i}}\right)=\iint_{S} b_{i}\left[\left|\frac{\nabla p_{i}}{\chi_{i}}\right|^{2}+\phi\left(b_{i}\right)\right] \mathrm{d} s \\
i=1,2, \ldots, I
\end{gathered}
$$

$M$ is the number of excitation sources, $N$ is the number of receivers for each source, $K_{m}\left(\mathbf{r}_{n}^{r}, t\right)$ is a weighting function, $\mathbf{r}_{n}^{r}$ is the position vector of the $n$th receiver, $t$ is time, $S$ is the area of reconstruction region, $I$ is the number of parameters to be reconstructed, $\tilde{\mathbf{v}}_{m}\left(\mathbf{r}_{n}^{r}, t\right)$ is the observed data at $\mathbf{r}_{n}^{r}$ corresponding to the $m$ th source, $\mathbf{v}_{m}\left(\mathbf{p} ; \mathbf{r}_{n}^{r}, t\right)$ is the calculated data for the $m$ th source using the guessed parameter $\mathbf{p}$. For the case of seismic inversion, $\mathbf{v}_{m}\left(\mathbf{p} ; \mathbf{r}_{n}^{r}, t\right)$ are pressure or particle velocity components, and $\mathbf{p}$ are bulk modulus, density and absorptive factor. For the case of electromagnetic inversion, $\mathbf{v}_{m}\left(\mathbf{p} ; \mathbf{r}_{n}^{r}, t\right)$ are electric or magnetic field components, and $\mathbf{p}$ consist of permittivity, magnetic permeability and conductivity. $\mathbf{p}$ is expressed as:

$$
\mathbf{p}=\left[\begin{array}{llll}
p_{1}(\mathbf{r}) & p_{2}(\mathbf{r}) & \cdots & p_{I}(\mathbf{r})
\end{array}\right]^{\mathrm{T}}
$$

$\mathbf{Q}_{2}(\mathbf{p}, \mathbf{b})$ is the gradient of the model parameters. It acts as a damping factor to control perturbation of error, and to increase the stability of solution. $\lambda_{i}$ and $\chi_{i}$ are regularization parameters. $\lambda_{i}$ is a smoothing parameter used to balance the weight of the data and the prior term. $\chi_{i}$ is a threshold parameter used to determine the preserved and smoothed gradient values. This parameter plays a key role in edge preservation when using Eq. (1) (Charbonnier et al, 1997).

This paper uses the potential function proposed by Geman and McClure (1985):

$$
\varphi(x)=\frac{x^{2}}{1+x^{2}}
$$

Substituting Eq. (5) into Eq. (3) gives:

$$
\phi\left(b_{i}\right)=b_{i}-2 \sqrt{b_{i}}+1
$$

where, $b_{i}$ is an auxiliary variable introduced in the halfsquare regularization method by Geman and Yang (1995). The introduction of this variable can simplify the calculation. Through derivation, if $b_{i}$ is given by:

$$
b_{i}=\varphi^{\prime}\left(\left|\frac{\nabla p_{i}}{\chi_{i}}\right|\right)=\left[1+\left|\frac{\nabla p_{i}}{\chi_{i}}\right|^{2}\right]^{-2}
$$

Eq. (3) reaches its minimum.

\section{Theoretical analysis}

We try to find the rule to determine regularization parameters by theoretical analysis and numerical simulation tests. The effect of the edge-preserving regularization parameters on reconstruction results, and the relation between the regularization parameters and the error of data will be discussed.

The edge-preserving regularization method used in this paper is different from the traditional Tikhonov regularization method. In the edge-preserving regularization method, the prior weight term relates to not only the regularization parameter $\lambda$, but also the choice of potential function and the regularization parameter $\chi$. From Eq. (3) it is seen that the prior term is a function of $\chi$, so it is impossible to determine the weight of the prior term only from $\lambda$.

Define an auxiliary parameter $\boldsymbol{\theta}$ :

$$
\theta_{i}=Q_{1} / \lambda_{i} Q_{2}
$$

From Eq. (8) it is known that $\boldsymbol{\theta}$ is a function of $\boldsymbol{\lambda}$ and $\boldsymbol{\chi}$, and it plays a role to balance the weight between the data and the 
prior term. It is clear that the function of $\boldsymbol{\theta}$ is similar to that of the regularization parameter $\lambda$ in the traditional Tikhonov regularization method. Once $\boldsymbol{\chi}$ and $\boldsymbol{\theta}$ are determined, $\lambda$ is determined uniquely too. Therefore, we actually determine the relationship of the regularization parameter $\chi$ and auxiliary parameter $\boldsymbol{\theta}$ with other factors.

According to the prior analysis and deviation theory, the auxiliary parameter $\boldsymbol{\theta}$ is a function of the error of observed data $\boldsymbol{\delta}$ :

$$
\boldsymbol{\theta}=f(\boldsymbol{\delta})
$$

The auxiliary parameter $\boldsymbol{\theta}$, which balances the data and the prior term, replaces the original regularization parameter $\lambda$. If the error of data $\boldsymbol{\delta}$ is large, $\boldsymbol{\theta}$ increases to enlarge the weight of the prior term. On the contrary, if $\boldsymbol{\delta}$ is small, $\boldsymbol{\theta}$ decreases to increase the weight of the data term.

There is another advantage to replacing $\boldsymbol{\lambda}$ by $\boldsymbol{\theta}$. Conventionally $\lambda$ is assigned a fixed value in the course of iteration. However, the difference between the observed and guessed data becomes small with iteration, so it is necessary to adjust the value of $\lambda$ in order to keep the functions of the data and prior term. If $\lambda$ is replaced by $\boldsymbol{\theta}, \boldsymbol{\theta}$ can be assigned a fixed value. Its meaning is that the ratio of the data and prior term keeps a fixed value. As a result, the roles of the data and prior term can be balanced. Obviously, it is much easier than to adjust $\lambda$.

The reason why the edge-preserving regularization method has the effect to protect the boundaries is the introduction of the regularization parameter $\chi . \chi$ is a threshold value used to determine which part of the gradient needs to be protected and which part needs to be smoothed. For simplicity, it is supposed that a single model parameter is reconstructed in this paper. Shown in Fig. 1 is the relation of the potential function $\varphi$ with $\chi$ and the gradient $|\nabla p|$.

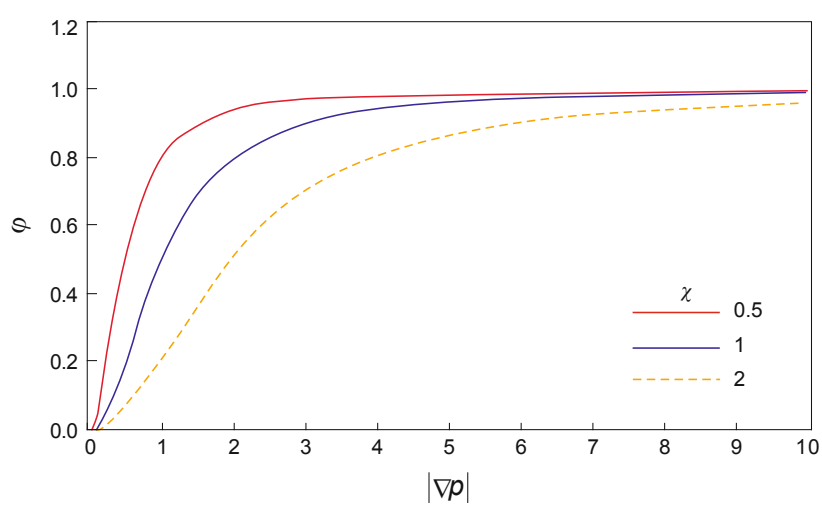

Fig. 1 Relation of $\varphi$ with $\chi$ and $|\nabla p|$

From Fig. 1 it is seen that the curve consists of two variable sections, one is on the left with a large slope, and the other is on the right with a small slope, and $\chi$ controls the position of the boundary between the two sections. Eq. (5) indicates that the potential function is monotonously increasing and has a horizontal asymptote. Its value is in the range of $[0,1]$. The gradient located in the left section is smoothed and that in the right section is protected.

If $\chi$ tends to 0 , the length of the left section tends to 0 too, and the value of the potential function is 1 for all $|\nabla p|$. In this case the prior term is of no effect, and the reconstruction result is equivalent to the least-square solution. With the increase of $\chi$, the length of the left section increases. If $\chi$ is large enough, the relation between the potential function and the gradient of the model $|\nabla p|$ is approximately linear. The gradients located in this linear part are smoothed identically. In this case the edges are not protected either. As a result, $\chi$ should be selected to let the gradient of the actual model be located (and be protected) in the right section, and to let the gradient created by error and noise be located (and be smoothed) in the left section.

\section{Influence of regularization parameters on reconstruction results}

It is necessary to understand how the regularization parameters influence the reconstruction results in order to know the relation between the regularization parameters and error of data.

Signal to noise ratio (SNR) is defined by

$$
\mathrm{SNR}=10 \log \frac{E_{S}}{E_{N}}
$$

where $E_{\mathrm{S}}$ and $E_{\mathrm{N}}$ is the energy of signal and noise, respectively. The noise in this paper is Gaussian.

$\chi$ is a particular parameter of edge-preserving regularization. The edge-preserving regularization method can protect boundaries because of this parameter. The influence of $\chi$ on the reconstruction result is complicated. It is essential to learn how it affects the reconstruction result from numerical examples.

The reconstruction of permittivity from an electric field is illustrated here as a numerical example. In the numerical example, the medium is nonmagnetic and only permittivity is reconstructed. As shown in Fig. 2(a) there are two buried objects with relative permittivity of 4 in a background with relative permittivity of 6 . The numerals in the $x$ and $z$ directions represent grid number. The grid length is $1.2 \mathrm{~cm}$ in both directions. The temporal interval is $0.02774 \mathrm{~ns}$. The excitation pulse is Gaussian, and its highest frequency is 1.0 $\mathrm{GHz}$. The size of the finite-difference time-domain (FDTD) simulation region is $161 \times 61$ nodes, and the duration of the data is 369 temporal intervals. The reconstruction region is from the 31 st to 131 st node in the $x$ direction and from the 7 th to 44 th node in the $z$ direction. In this example there are 9 excitation sources and 19 receivers for each source. The relative position of a source and its receivers are fixed for all excitations. The gap between the source and the first receiver is two grids, the interval of receivers is one grid, and the interval of source points is ten grids. In Fig. 2(a) the circles on the top of the model indicate the positions of sources. The initial guess of permittivity of the reconstruction region is that of the background. 
Fig. 2(b) is the reconstructed result after 100 iterations from noisy data with $\mathrm{SNR}=10 \mathrm{~dB}$ by using the reconstruction method without regularization. Fig. 2(c)-(g) are the reconstructed results after 100 iterations from the noisy data by using the edge-preserving regularization method when $\theta=0.5, \chi=10^{-7}, 0.05,0.1,5,10$, respectively.

By comparing Fig. 2(c) $\left(\chi=10^{-7}\right)$ and Fig. 2(b), it is known that they are almost the same. Since $\chi$ is very small, the prior term is of no effect, and the reconstructed result is the result of the conventional least-square solution. Fig. 2(d) is the result for $\chi=0.05$, where the gradients of the model and noise are both located in the right gentle section. Since both gradients are protected, the reconstructed result is too smooth, and the boundaries of the model are out of focus. The result is a little far from the actual model. Fig. 2(e) is the result for $\chi=0.1$ where the left section gets longer and the gradients due to the model and due to noise are separated in the left and right sections respectively. The gradient due to noise is located in the left section, and the gradient due to the model is located in the right section. Therefore, the edges of the model are protected and most of the noise is suppressed. Only the high amplitude noise affects the reconstructed image in the form of spots. Fig. 2(f) is the result for $\chi=5$ where the left section gets much longer and the gradients due to the model and due to noise are both located in the left section. Both gradients are weakened and the gradient of the model is more weakened, so the noisy spots are more evident. Fig. $2(\mathrm{~g})$ is the result after 63 iterations for $\chi=10$ where the left section gets longer so that the whole left section is approximately linear. For this case both gradients are smoothed and the reconstructed result is smooth like Fig. 2(d). With the increase of $\chi$, the protected part of gradient gets small, and the reconstructed result is close to the initial guess.

Table 1 illustrates the influence of the regularization parameter $\chi$ on the reconstructed result. The influence of the auxiliary parameter $\theta$ on the reconstructed result is obvious. With the increase of $\theta$, the weight of the prior term gets large, and the reconstructed result gets smooth. Conversely, with the decrease of $\theta$, the weight of the data term gets large, and the influence of noise becomes evident.

Table 1 Influence of regularization parameter $\chi$ on reconstructed result

\begin{tabular}{cccccc}
\hline$\chi$ & $10^{-7}$ & 0.05 & 0.1 & 5 & 10 \\
Ability to suppress noise & Almost none & Good & Ordinary & Ordinary & Good \\
Protection of boundaries & Almost none & Slight & Obvious & Slight & Slight \\
Inversion result & Influenced by noise & Too smooth & Best & $\begin{array}{c}\text { Unclear edges, } \\
\text { too many noisy spots }\end{array}$ & Too smooth \\
\hline
\end{tabular}

From the theoretical analysis and numerical tests, the optimal parameters for the edge-preserving regularization method are $\theta=0.3$ and $\chi=0.1$. With these parameters the reconstructed result is shown in Fig. 2(h). Comparing Fig. 2(h) with 2(b), it is observed that the influence of noise is greatly weakened, and the edges are protected. Fig. 2(h) is similar to the reconstruction result shown in Fig. 2(i) from noise free data.

The auxiliary parameter $\theta$ has a relatively simple effect on reconstruction than the regularization parameter $\chi$. As a result, in practice $\theta$ is roughly determined at first, then $\chi$ is adjusted.

\section{Relation between regularization parameters and error of data}

Fig. 3(a) and 3(b) are the reconstructed results after 100 iterations from noisy data with $\mathrm{SNR}=20 \mathrm{~dB}$ by using the reconstruction method without and with edge-preserving regularization. Fig. 3(b) is the best result by testing time after time, the regularization parameters are $\theta=0.1$ and $\chi=0.08$. It is clear that in Fig. 3(b) the influence of noise on the reconstructed result is weak. However, the reconstructed value is not so good, and the edges are not protected quite well. From this example it is deduced that the application of the edge-preserving regularization is not so meaningful for the case of $\mathrm{SNR}>20 \mathrm{~dB}$.

Fig. 3(c) and 3(d) are the reconstructed results after 100 iterations from noisy data with $\mathrm{SNR}=15 \mathrm{~dB}$ by using the reconstruction method without and with edge-preserving regularization. Fig. 3(d) is the best result by testing time after time, the regularization parameters are $\theta=0.2$ and $\chi=0.09$. It is clear from Fig. 3(c) and 3(d) that the effect of the edgepreserving regularization is evident. The background of Fig. 3(d) is less affected by noise and the boundaries are much clearer than those of Fig. 3(c).

Fig. 3(e), (f) and (g) are the reconstructed results after 100 iterations from noisy data with $\mathrm{SNR}=5 \mathrm{~dB}$ by using the reconstruction method without and with edge-preserving regularization. Fig. 3(f) is the result for the regularization parameters $\theta=0.3$ and $\chi=0.2$, and Fig. $3(\mathrm{~g})$ is the result for the regularization parameters $\theta=0.35$ and $\chi=0.15$. Fig. 3(f) and $3(\mathrm{~g})$ are better than Fig. 3(e). It is almost impossible from Fig. 3(e) to recognize the two objects due to the influence of noise. With the increase of iterations the image becomes even worse. It is noted that with the increase of noise, the conventional reconstruction method fails to reconstruct the model. From the edge-preserving method, a stable reconstruction result can 


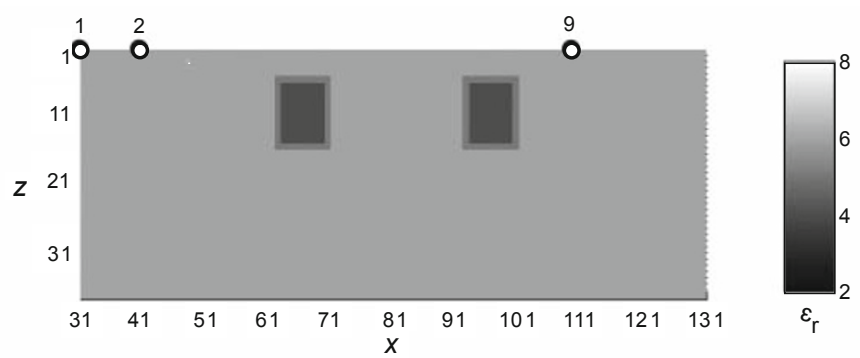

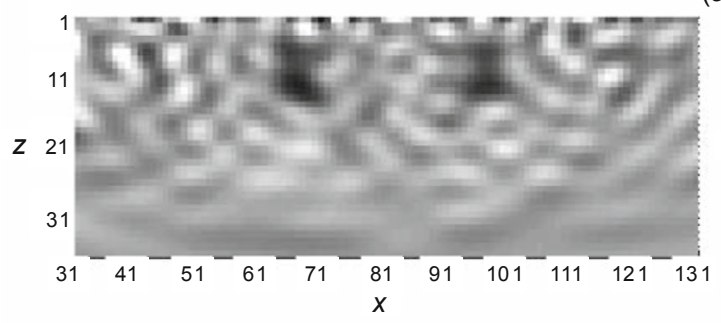

(b)

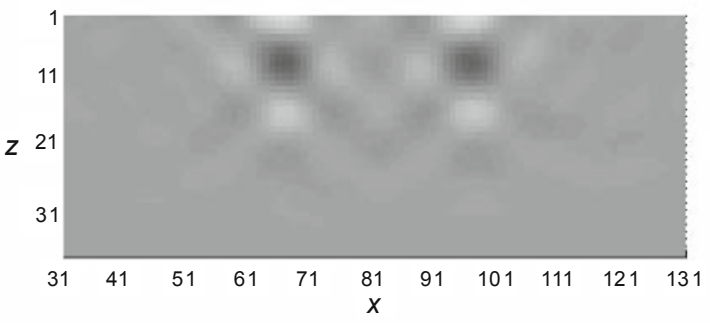

(d)

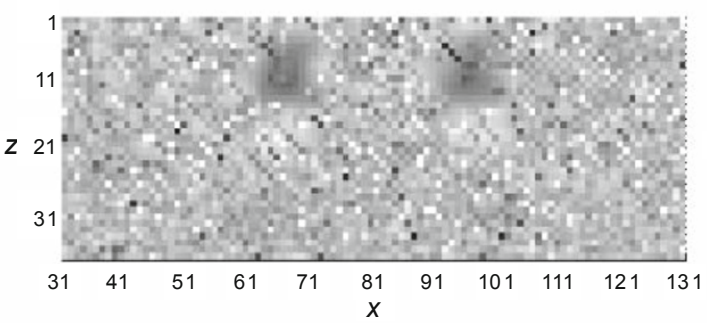

(f)

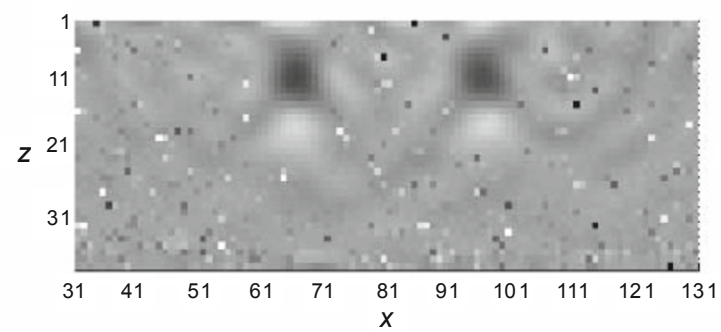

(h)

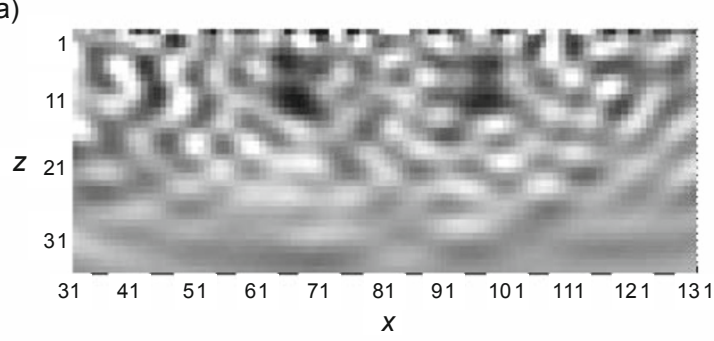

(c)

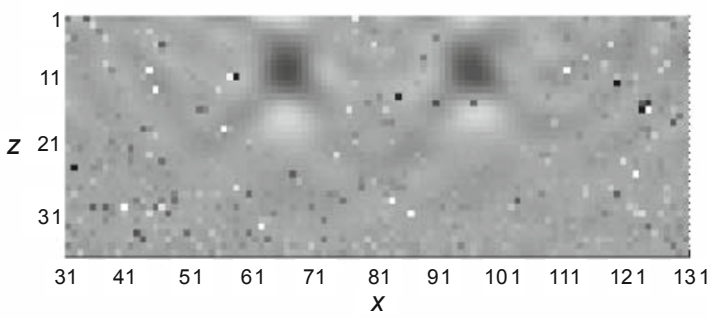

(e)

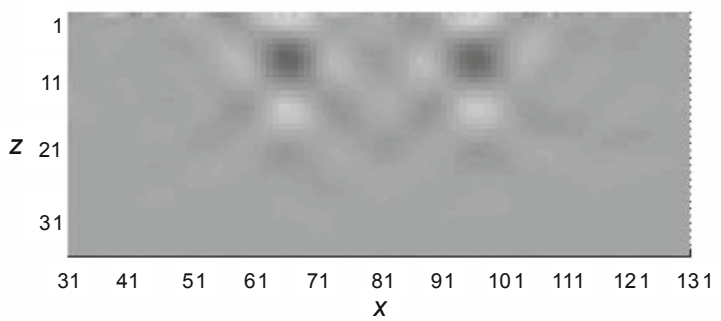

(g)

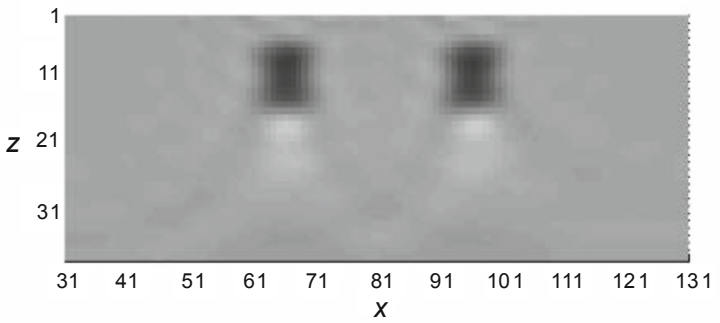

(i)

Fig. 2 Actual distribution of relative permittivity in the reconstruction region (a). Reconstructed images after 100 iterations from the data with $\mathrm{SNR}=10 \mathrm{~dB}$ using non-regularization method (b), using edge-preserving regularization method for $\theta=0.5, \chi=10^{-7}$ (c), $\theta=0.5$, $\chi=0.05$ (d), $\theta=0.5, \chi=0.1$ (e), $\theta=0.5, \chi=5$ (f), $\theta=0.5, \chi=10$ (after 63 iterations) (g), $\theta=0.3, \chi=0.1$ (h). Reconstructed image after 100 iterations from noise free data using non-regularization method (i)

be obtained. Fig. 3(f) for $\theta=0.3$ and $\chi=0.2$ is more affected by noise than Fig. $3(\mathrm{~g})$ for $\theta=0.35$ and $\chi=0.15$. However, the boundaries in Fig. 3(f) are protected much better and the value of permittivity is more accurate than that in Fig. 3(g).

Fig. 3(h), (i) and (j) are the reconstructed results after 100 iterations from noisy data with $\mathrm{SNR}=0 \mathrm{~dB}$ by using the reconstruction method without and with edge-preserving regularization. Fig. 3(i) is the result for the regularization parameters $\theta=0.4$ and $\chi=0.3$, and Fig. $3(\mathrm{j})$ is the result for the regularization parameters $\theta=0.4$ and $\chi=0.25$. The phenomena are almost the same as the case of $\mathrm{SNR}=5 \mathrm{~dB}$, but the influence of noise is more evident. It is clear that the edge-preserving regularization plays a very important role in suppressing noise and protecting boundaries. 


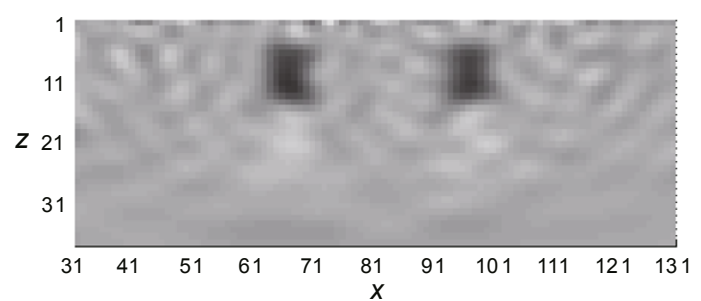

(a)

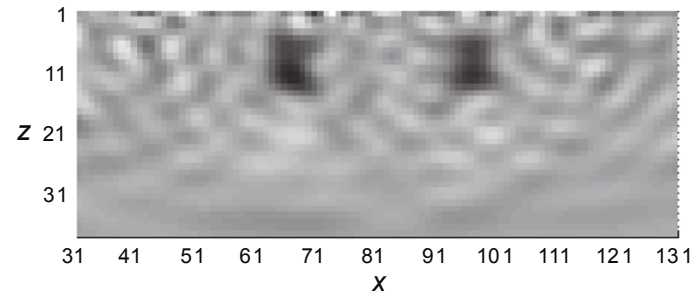

(c)

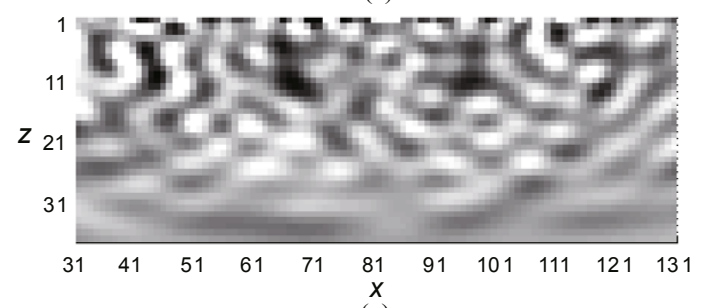

(e)

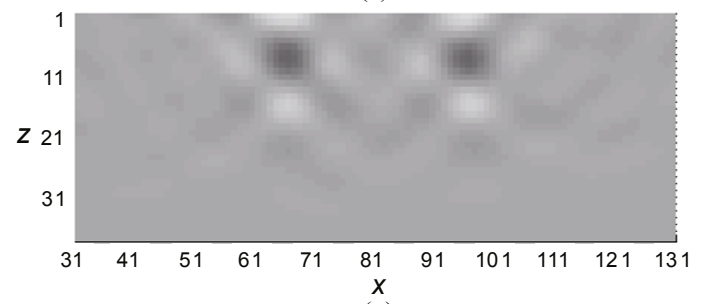

(g)

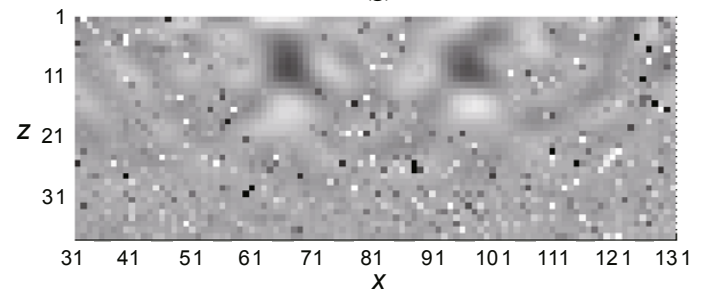

(i)

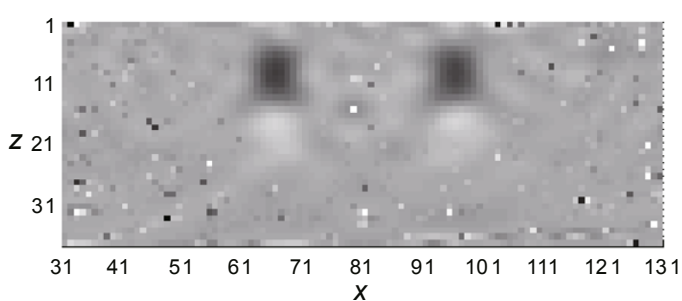

(b)

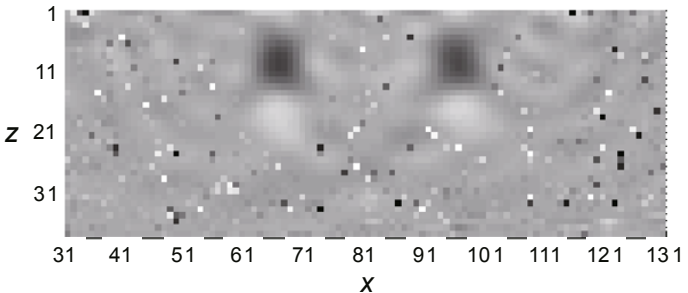

(d)

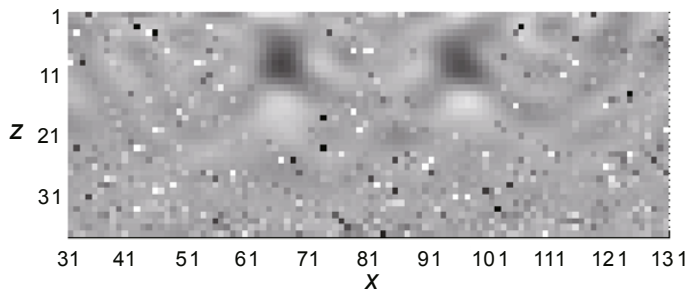

(f)

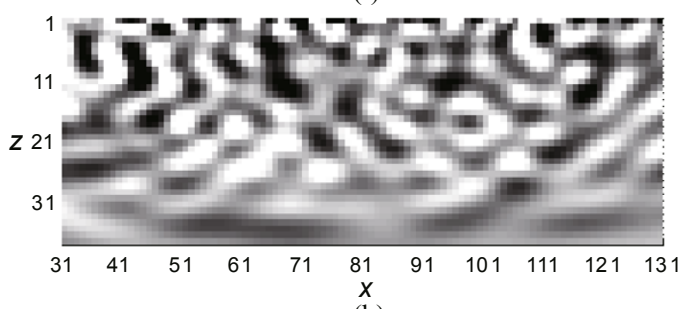

(h)

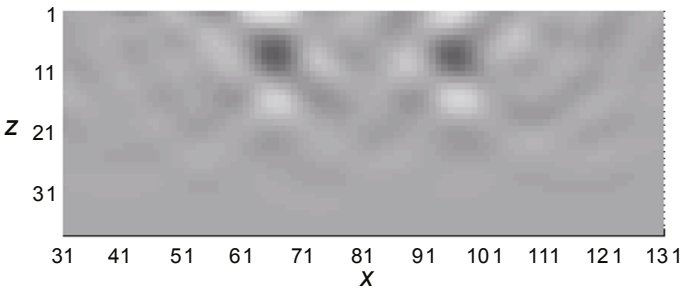

(j)

Fig. 3 Reconstructed image after 100 iterations from the data with $\mathrm{SNR}=20 \mathrm{~dB}$ using non-regularization method (a), using edge-preserving regularization method for $\theta=0.1, \chi=0.08$ (b); from the data with $\mathrm{SNR}=15 \mathrm{~dB}$ using nonregularization method (c), using edge-preserving regularization method for $\theta=0.2, \chi=0.09$ (d); from the data with $\mathrm{SNR}=5 \mathrm{~dB}$ using non-regularization method (e), using edge-preserving regularization method for $\theta=0.3, \chi=0.2$ (f), $\theta=0.35, \chi=0.15$ (g); from the data with $\mathrm{SNR}=0 \mathrm{~dB}$ using non-regularization method (h), using edge-preserving regularization method for $\theta=0.4, \chi=0.3$ (i), $\theta=0.4, \chi=0.25$ (j)

From the above numerical examples, the relation of optimal regularization parameters with SNR are listed in Table 2. By fitting, two graphs of $\theta$-SNR and $\chi-E_{\mathrm{N}} / E_{\mathrm{S}}$ are plotted in Fig. 4.

It shows that the relation of $\theta$ with SNR and $\chi$ with $E_{\mathrm{N}} / E_{\mathrm{S}}$ are nearly linear. Consequently it is possible to determine $\theta$ and $\chi$ by SNR. However, this paper does not give a regression equation since the regression coefficients of the equation are strongly related to the variance of the model, the initial guess of the model, the reconstruction method, and the type of noise. For example, if the initial model is very close to the actual one, $\theta$ should be large to emphasize the prior term. If the initial model is a little far from the actual one, $\theta$ should be small to emphasize the data term. If the variance of model is large, $\chi$ is increased to smooth the noise. However, the model variance and the approaching degree of initial guess are not estimated as easily as SNR, so the maneuverability is low. 
Table 2 Relation of optimal regularization parameters with SNR

\begin{tabular}{cccc}
\hline SNR, dB & $E_{\mathrm{N}} / E_{\mathrm{S}}$ & Auxiliary parameter $\theta$ & Regularization parameter $\chi$ \\
\hline 20 & 0.100 & 0.1 & 0.08 \\
15 & 0.178 & 0.2 & 0.09 \\
10 & 0.316 & 0.3 & $0.15-0.2$ \\
5 & 0.562 & $0.3-0.35$ & $0.25-0.3$ \\
\hline
\end{tabular}

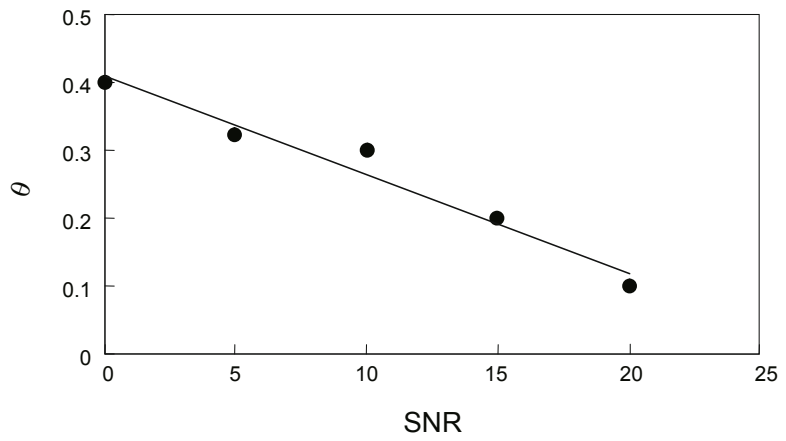

(a)

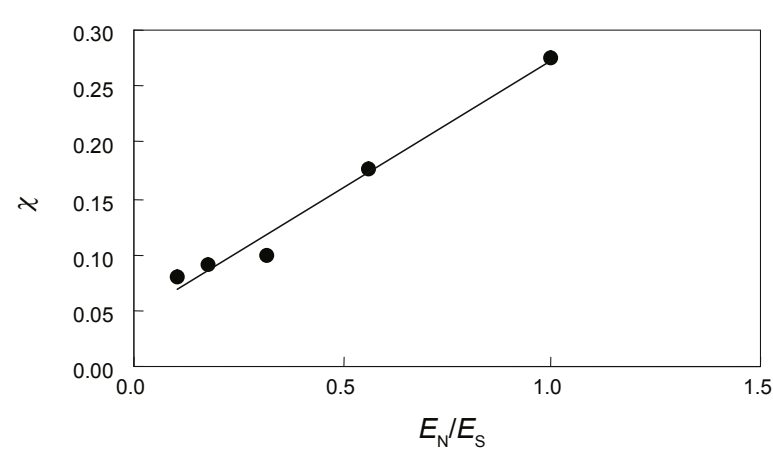

(b)

Fig. 4 Relation of auxiliary parameter $\theta$ with $\operatorname{SNR}\left(\right.$ a), and regularization parameter $\chi$ with $E_{\mathrm{N}} / E_{\mathrm{S}}$ (b)

\section{Conclusions}

In this paper the edge-preserving regularization method in the reconstruction of physical parameters is discussed. This method can provide a stable, edge-protected, and high resolution result. By theoretical analysis and numerical computation, we find the influence of the regularization parameters on the reconstruction results, and the relation between the parameters and the error of data. It is found that with the increase of the auxiliary parameter $\theta$, the weight of the prior term gets large, and the reconstructed result gets smooth; with the decrease of $\theta$, the weight of the data term gets large, and the influence of noise becomes evident. The relation of $\theta$ and regularization parameter $\chi$ with SNR are nearly linear. On this basis, a way to select regularization parameters is given to avoid the blindness and randomness in the applications.

The edge-preserving regularization method used in this paper always protects large gradients and smoothes small gradients. It is based on the assumption that larger gradients are the result of real boundaries, and small gradients are the result of the error of data such as noise. This assumption is not tenable for the case of low SNR. As a result, when the error of data is large, this regularization method should be used carefully. It is better to use other regularization methods and compare these results to judge the quality of the results. In addition, the rule to determine the regularization parameters obtained in this paper is qualitative. Experience and understanding of the regularization method are needed. As a result, this method needs to be researched further.

\section{Acknowledgements}

This work was supported in part by the National Natural Science Foundation of China under Grant-in-Aid 40574053, the Program for New Century Excellent Talents in University of China (NCET-06-0602), and the National 973 Key Basic Research Development Program (No.2007CB209601).

\section{References}

Charbonnier P, Blanc-Feraud L, Aubert G, et al. Deterministic edgepreserving regularization in computed imaging. IEEE Transactions on Image Processing. 1997. 6(2): 298-311

Ding S J and Jin X S. Solution properties of ill-posed problem in adjustment and resolution of regularization parameter. Science of Surveying and Mapping. 2006. 31(2): 22-24 (in Chinese)

Geman D and Yang C. Nonlinear image recovery with half-quadratic regularization and FFT's. IEEE Transactions on Image Processing. 1995. 4(7): 932-946

Geman S and McClure D E. Bayesian image analysis: An application to single photon emission tomography. Proceedings of the Statistical Computation Section, American Statistical Association. 1985. 1218

Guo X Z, Zhang X M and Xu L. Regularized strategy on the Gauss kernel. Journal of Hebei Normal University (Natural Science 
Edition). 2006. 30(2): 151-153 (in Chinese)

Han B and Fu Y H. A regularization homotopy method for solving nonlinear ill-posed problems. Journal of Natural Science of Heilongjiang University. 2005. 22(5): 659-663 (in Chinese)

Jin Q N and Hou Z Y. Optimal regularization parameter choice for iterated Tikhonov regularization. Applied Mathematics-A Journal of Chinese Universities. 1996. 11(3): 321-328 (in Chinese)

Kunisch K and Zou J. Iterative choices of regularization parameters in linear inverse problems. Inverse Problems. 1998. 14(5): 1247-1264

Liu D and Zhao R C. The method of preserving edge smoothing. Journal of Northwestern Polytechnical University. 2000. 18(1): 102105 (in Chinese)

Shen J S, Sun W B, Zhao W J, et al. A 2.5D cross-hole electromagnetic modelling and inversion method and its application to survey data from the Gudao Oilfield, east China. Journal of Geophysics and Engineering. 2008. 5(4): 401-411

Takenaka T, Jia H T and Tanaka T. Microwave imaging of electrical property distributions by a forward-backward time-stepping method. Journal of Electromagnetic Waves and Applications. 2000. 14(12): $1609-1626$

Takenaka T, Jia H T and Tanaka T. Microwave imaging of an anisotropic cylindrical object by a forward-backward time-stepping method. IEICE Transactions on Electronics. 2001. E84-C(12): 1910-1916

Wang P, Li G F, Zhang L Q, et al. Thin-layer reservoir prediction in the lower Es_1 of the western ramp of Qikou Depression. Journal of
China University of Petroleum (Edition of Natural Science). 2008. 32(2): 28-33 (in Chinese)

Wang S X, Li X Y, Qian Z P, et al. Physical modeling studies of 3-D P-wave seismic for fracture detection. Geophysical Journal International. 2007. 168(2): 745-756

Wei C, Li X F and Zhang M G. The geophysical inverse method based on Quantum Monte Carlo. Chinese Journal of Geophysics. 2008. 51(5): 1494-1502 (in Chinese)

Yang P J, Yin X Y and Zhang X. Poststack seismic blind inversion and application. Oil Geophysical Prospecting. 2008. 43(3): 284-290 (in Chinese)

Zhang H B, Shang Z P, Yang C C, et al. Estimation of regular parameters for the impedance inversion. Chinese Journal of Geophysics. 2005. 48(1): 181-188 (in Chinese)

Zhang H B and Yang C C. A constrained impedance inversion method controlled by regularized parameters. Chinese Journal of Geophysics. 2003. 46(6): 827-834 (in Chinese)

Zhou H, Sato M, Takenaka T, et al. Reconstruction from antennatransformed radar data using a time-domain reconstruction method. IEEE Transactions on Geoscience and Remote Sensing. 2007. 45(3): 689-696

Zou X W, Wang B E, Kou T, et al. A new method of choosing regularization parameters based on genetic algorithm. Science and Technology Review. 2006. 24(2): 46-49 (in Chinese)

(Edited by Hao Jie) 